\title{
An ICSC Model for Detecting Human Skin in JPEG Images
}

\author{
HANI. K. ALMOHAIR, \\ Computer and Information Technology \\ King Fahd University of Petroleum and Minerals, 31261, KFUPM \\ Dhahran, Eastern Region, SAUDI ARABIA
}

\begin{abstract}
The detection of human skin color has proven to be a useful and robust technique for detecting nude images, face detection, localization and tracking. This paper presents an Improved Chromatic Skin Color model to detect the human skin in JPEG images; the ICSC model detected the human skin with detection rates more than 90\%. A threshold method and 2D Gaussian model will improve the accuracy of skin regions detected
\end{abstract}

Keywords - Skin color models, Pixels classification, Skin detection

Received: December 6, 2020. Revised: April 14, 2020. Accepted: April 21, 2020. Published: April 30, 2020.

\section{Introduction}

This image segmentation is a process of dividing an image into non-overlapping regions consisting of groups of connected homogeneous pixels. Typical parameters that define the homogeneity of a region in a segmentation process are color, depth of layers, gray levels, texture, etc [1]. A good example of image segmentation is skin detection, which is achieved by classifying the image pixels into two groups; skin pixels and non-skin pixels using skin color information. The process of utilizing skin color information as a cue in skin detection has gained much attention because skin color provides computationally effective yet, robust information against rotations, scaling and partial occlusions [2]. Skin color detection is primarily an important process in applications such as face detection [3-5], gesture analysis [6], Internet pornographic image filtering [7], surveillance systems [8-12]. In order to make use of skin color information, many researches have been directed to understanding its characteristics. Research analysis has shown that human skin color has a restricted range of hues and is not deeply saturated, as the appearance of skin is formed by a combination of blood (red) and melanin (brown, yellow) [13].

This work was supported by King Fahd University of Petroleum and Minerals (KFUPM)
Therefore, human skin color does not fall randomly in a given color space, but rather clustered within a small area in the color space. Several studies have shown that the major difference in skin color among different people lies largely in their intensity rather than in their chrominance [14]. Thus, if an image is first converted into a color space, which provides a separation of luminance channel and two chrominance components like the normalized ( $\mathrm{r}, \mathrm{g}, \mathrm{b})$ color space, then skin-like regions can easily be detected [15]. Research has been performed on the detection of human skin pixels in color images and on the discrimination between skin and nonskin pixels by use of various statistical color models. Some researchers have used skin color models such as Gaussian, Gaussian mixture of histograms $[16,17]$. New technique for skin color detection by estimating the distribution of skin and non-skin pixels in the color space using labeled training data is discussed in [18]. In this paper we suggest strongly the Improved Chromatic Skin Color model (ICSC) for two fundamental reasons; it can be easily constructed form training data as well as it has been proven that it's performance can reach over than $90 \%$. The paper is organized as follows. Section 2 explains the ICSC model. Section 3 describes the threshold technique. The results and discussion is given in section 4 . Section 5 discusses the conclusion. 


\section{ICSC Model}

The various color spaces can be easily converted to RGB representation, however, the RGB is not necessary for characterizing skin color since it is not the best color representation in the color spaces. A triple [r,g,b] doesn't represent only color but also brightness, due to that property it was used to represent skin color in there chromatic color space. Chromatic colors (r,g) [4, 19], known as "pure " colors in the absence of brightness, are defined by:

$$
\begin{gathered}
\left\lfloor\begin{array}{l}
r \\
g \\
b
\end{array}\right\rfloor=\left\lfloor\begin{array}{ccc}
1 /(R+G+B) & 0 & 0 \\
0 & 1 /(R+G+B) & 0 \\
0 & 0 & 1 /(R+G+B)
\end{array}\right\rfloor\left\lfloor\left\lfloor\begin{array}{l}
R \\
G \\
B
\end{array}\right\rfloor\right. \\
\quad r=\frac{R}{R+G+B} \quad, \quad g=\frac{G}{R+G+B}
\end{gathered}
$$

Color blue is redundant after the normalization because

$\mathrm{r}+\mathrm{g}+\mathrm{b}=1$.

A color histogram is the distribution of colors in the color space; Figure (1-A) shows a various people's images, and corresponding area for histogram analysis shown in Figure (1-B). The histogram of the skin-color selected is illustrated in Figure (1-C). The color distribution of the skin color is clustered in small area of the chromatic color space, skin colors of different people are very close but they differ mainly in intensities. Figure 1 and 2 show a skin color distribution and clustering of fifty people with different skin colors in the chromatic color space, the distribution was obtained by analyzing skin of different races, including Asian, African, American, Arabic and Caucasian.
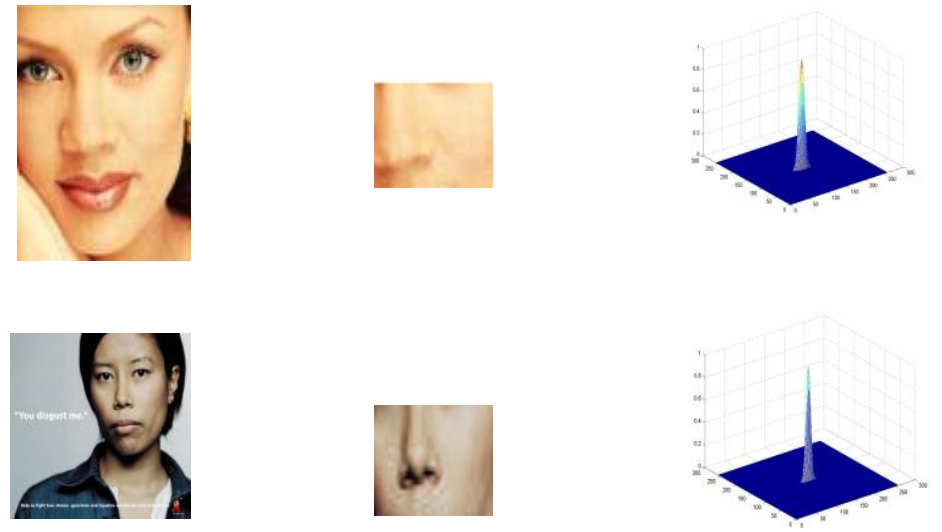

(1)
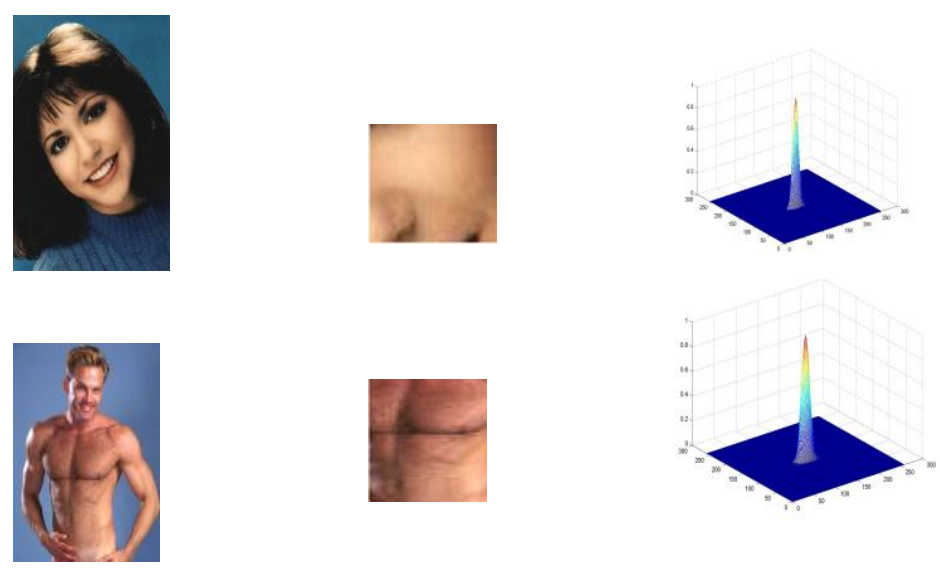

(A)

(B)

(C)
Figure1: Various people images (A), skin area for histogram analysis (B), histogram for selected skin area (C)

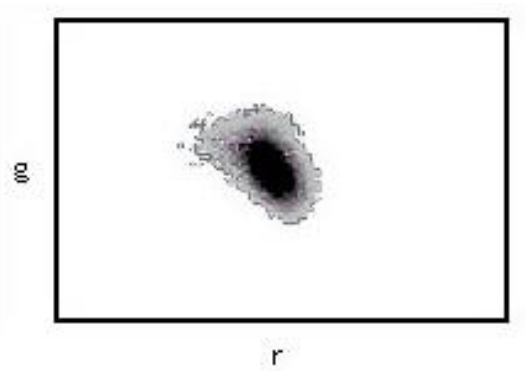

Figure2: Cluster of Skin color distribution. 
The skin color distribution can be represented by $2 \mathrm{D}$ Gaussian model by substituting the mean and covariance of the color distribution in chromatic color space into the Gaussian distribution model. Where mean and covariance values can be easily estimated by using equations 3 and 4 .

$$
\begin{aligned}
r^{-} & =\frac{1}{N} \sum_{i=1}^{N} r_{i} \\
g^{-} & =\frac{1}{N} \sum_{i=1}^{N} g_{i} \\
\sigma_{g}^{2} & =\frac{1}{N}\left(g_{i}-g^{-}\right)^{2} \\
\sigma_{r}^{2} & =\frac{1}{N}\left(r_{i}-r^{-}\right)^{2}
\end{aligned}
$$

By using the adaptive threshold method, we can classify the skin and non-skin pixels, where the skin pixels represented with the white color.

\section{Threshold Technique}

The final step in the detection of human skin in JPEG images is creating a skin-segmented image by using a threshold value of probability. If the probability of a pixels in the input images (image under test) is more or equal to the estimated threshold value. It would be supposed that this pixel represents skin color, if it doesn't, then it would be supposed that it doesn't represent skin color. The skin color pixels are white and the other ones are black in skin-segmented image, the threshold value used in skin-segmented image is called an optimal threshold value, where the minimum increase in region size is observed while decreasing the threshold value [20]. As a suitable compromise to discriminate between the skin and non-skin pixels, an optimal threshold value should be obtained. It is obtained through the proportion of the true positive TP over the ensemble of regions of skin, which is approximately equal to the proportion of true negative $\mathrm{TN}$ over the ensemble of regions when that proportion doesn't contain skin equivalently when the proportion of false negative FN equals the proportion of false positive FP [21]. Since this method is better for illumination variations, and for different skin colors, pixel considered as skin if the value is greater than the estimated optimal threshold value.
In this model, the threshold value selected start from 1 and decremented the threshold value in steps of 0.1 until 0.0 is reached. At each threshold value the TP and FN are determined and applied to the formula $\mathrm{TP}+\mathrm{FN}=1$. If true positive values plus false negative values are equal approximately to one, this value is considered as the optimal threshold value. The optimal threshold value was determined by a conducted test designed for testing the data images to prove the formula $\mathrm{TP}+\mathrm{FN}=1$. The testing data includes many images of different humans of different races. Besides, the skin and non-skin pixels are classified according to each image. Figure 3 shows how the optimal threshold value in our model was determined.

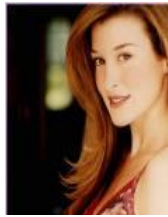

$$
\text { Original }
$$$$
\text { image }
$$

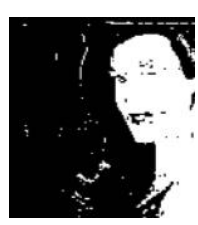

(B)

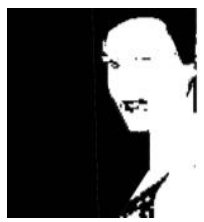

(A)

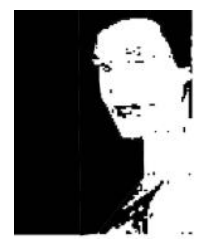

(C)
Figure 3: first column the original image, real skin pixels (A), real and non-real skin pixels detected (B), and the real skin pixels detected (C).

Table 1 shows the number of the skin and non-skin pixels for all the above cases

\begin{tabular}{|c|c|}
\hline Real skin pixels (A) & 128557 \\
\hline $\begin{array}{c}\text { Real and non-real skin } \\
\text { pixels detected (B) } \\
\text { Real skin pixels detected } \\
\text { (C) }\end{array}$ & 128509 \\
\hline TP & 125945 \\
\hline FP & 0.979 \\
\hline FN & 0.019 \\
\hline
\end{tabular}

Table 1: Number of real and non-real skin pixels detected 
From the table above, the TP and FN were estimated. The TP is recognized from the proportion of pixels detected as skin which are really skin pixels to the total number of real skin pixels, while the FN identified from the proportion of pixels detected as skin which are not real skin pixels to the total number of real skin pixels. It is wroth mentioning that these TP and $\mathrm{FN}$ values prove the formula $\mathrm{TP}+\mathrm{FN}=1$.

The obtained optimal threshold value is equal to 0.1 . This optimal value is estimated after a conducted test for the training data with different threshold values. From the table above we found $\mathrm{TP}+\mathrm{FN}=0.979+0.02=$ 0.999

The procedure for creating the ICSC model is as follows:

1. Collect a set of JPEG images of different people from the Internet or from any available images database.

2. Select manually the sample skin colored region from each image, this resulted in big number of the skin colored pixels.

3. Create a color histogram of the samples selected.

4. Estimate the mean and covariance of the color distribution in chromatic color-space based on mean and covariance functions.

5. Substitute the estimated parameters into the $2 \mathrm{D}$ Gaussian distribution model.

6. By using the adaptive threshold method, the skin and non-skin pixels are classified, where the skin pixels represented with the white color.

\section{Results and discussion}

Improved Chromatic Skin Color model (ICSC) is applied to a set of testing data images of more than fifty images download from the Internet, belonging to people of different races, The Accuracy of the (ICSC) used to detect human skin in JPEG images is measured by the Correct Detection ratio (CDR) and False detection Ratio (FDR). Where the CDR defined as the proportion of pixels detected as skin, which are really skin pixels to the total number of real skin pixels, and the FDR is the proportion of pixels detected as skin which are not real skin pixels to the total number of real skin pixels. Figure 4 shows some images and the skin detection regions, the detection rates reached more than $90 \%$. The non-skin images results are shown in Figure 5. Images that contain luminance are also detected correctly, but with detection rates more than 80 and less than $90 \%$ as illustrated in Figure 6.
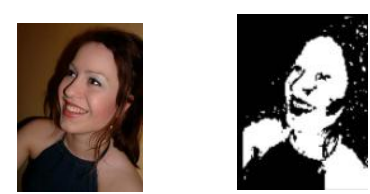

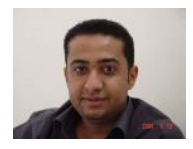

(A)

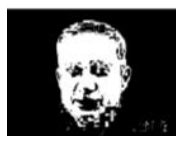

(B)
Figure 4: Original images (A) and the skin region detected (B)
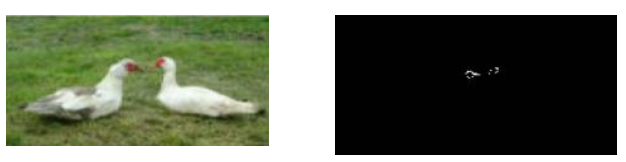

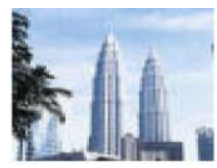

(A)

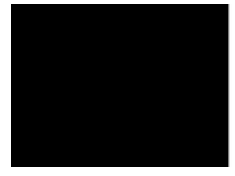

(B)
Figure 5: Original Non-skin Images (A) and skin region detected (B)

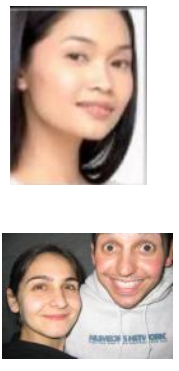

(A)
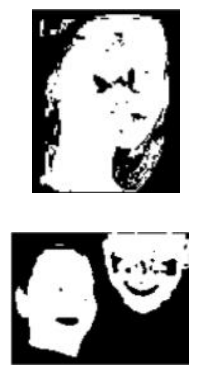

(B)
Figure 6: Original images that contain luminance (A) and skin region detected (B) 


\section{Conclusion}

In this paper, an Improved Chromatic Skin Color model (ICSC) is proposed and implemented successfully for detecting human skin in JPEG images, Since ICSC model needs to classify skin and non-skin pixels, the adaptive threshold method has been improved the accuracy of the skin region detected effectively. ICSC model has better performance for detection rates above 90\% (Figure7). This result shows that our model is more appropriate to avoid false detection areas while still remaining a high degree of correct detection. It has been found that the more luminance in images, the less detection ratio (Figure 8).

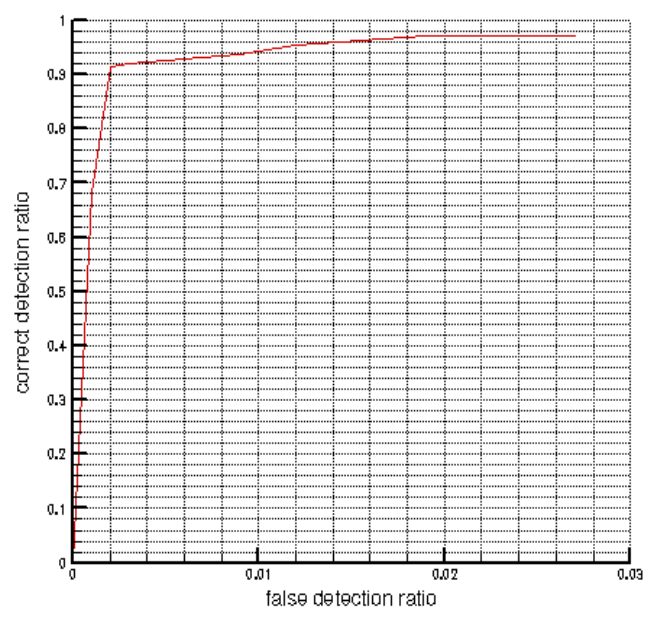

Figure 7: Skin detection rates using ICSC model (More than 90\%)

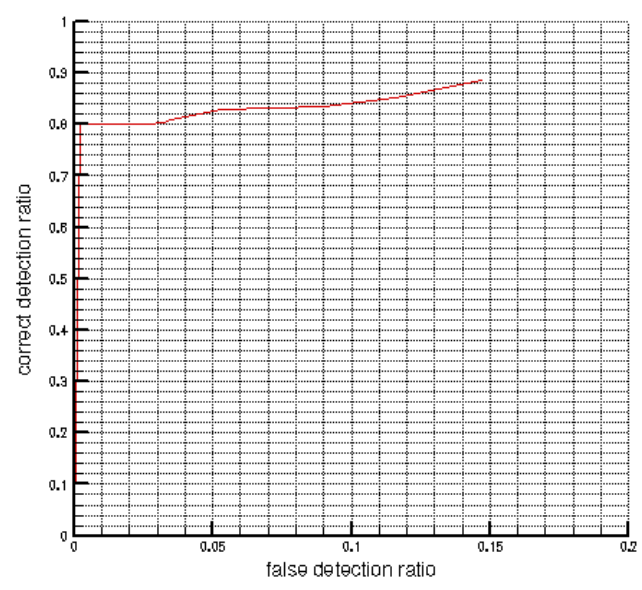

Figure 8: Skin detection rates for luminance images (More than 80\% and less than 90\%)

\section{ACKNOWLEDGMENT}

The Author Would Like To Acknowledge The Support Provided By The Deanship Of Scientific Research (Dsr) At King Fahd University Of Petroleum \& Minerals (Kfupm) For Funding This Work

\section{REFERENCES}

[1] C. C. Liu and P. C. Chung, Objects extraction algorithm of color image using adaptive forecasting filters created automatically, International Journal of Innovative Computing, Information and Control, vol.7, no.10, pp.57715787, 2011.

[2] P. Kakumanu, , S. Makrogiannis, and N. Bourbakis, A survey of skin-color modeling and detection methods, Pattern Recognition, vol.40, no.3, pp.1106-1122, 2007.

[3] C. Zhipeng, H. Junda and Z. Wenbin, Face detection system based on skin color model, 2nd International Conference on Networking and Digital Society (ICNDS), vol.2, pp.664-667, 2010.

[4] J. M. Chaves-Gonzalez, M. A. Vega-Rodriguez, J. A. Gomez-Pulido and J. M. Sanchez-Perez, Detecting skin in face recognition systems: A colour spaces study, Digital Signal Processing, vol.20, no.3, pp.806-823, 2010.

[5] Z. Zakaria, N. A. Isa and S. A. Suandi, Combining Skin Colour and Neural Network for Multiface Detection in Static Images, Symposium on Information \& Communication Technology (SPICT09), Kuala Lumpur, pp.147154, 2009.

[6] J. Han, G. Awad and A. Sutherland, Automatic skin segmentation and tracking in sign language recognition, Computer Vision, IET, vol.3, no.1, pp.24-35, 2009.

[7] J. S. Lee, Y. M. Kuo and P. C. Chung, Detecting Nakedness in Color Images. Intelligent Multimedia Analysis for Security Applications, Springer Berlin, Heidelberg SCI 282, pp. 225236, 2010.

[8] Z. Zhang, H. Gunes, and M. Piccardi, Head detection for video surveillance based on categorical hair and skin colour models, 16th 
IEEE International Conference on Image Processing (ICIP), pp.1137-1140, 2009.

[9] Yen H. C, Kai T. H. and Shanq J. R, Statistical skin color detection method without color transformation for real-time surveillance systems, Journal Engineering Applications of Artificial Intelligence, Volume 25 Issue 7, pp. 1331-1337October, 2012.

[10] nils janssen and neil robertson, on the detection of low-resolution skin regions in surveillance images, the eighth international workshop on visual surveillance - vs2008, 2008.

[11] Y. M. Mustafah, T. Shan a, A. W. Azman, A. Bigdeli and C. Lovell, Real-Time Face Detection and Tracking for High Resolution Smart Camera System, Digital Image Computing Techniques and Applications, 2007.

[12] Megha Sharma,Seema Verma, A S Mandal, Block Based Skin Color Detection for Automated Video Surveillance System, International Journal of Scientific \& Engineering Research, Volume 3, Issue 11, November-2012

[13] M. M. Fleck, D. A. Forsyth and C. Bregler, Finding Naked People, 4th European Conference on Computer Vision, Cambridge, UK, pp.593-602, 1996.

[15] J. Yang and A. Waibel, A real-time face tracker, Proceedings of the 3rd IEEE Workshop on Applications of Computer Vision (WACV '96), pp.142-147, 1996.

[16] L. Chen, J. Zhou, Z. Lid, W. Chen and G. Xiong, A skin detector based on neural network, IEEE International Conference on Communications, Circuits and Systems, vol.1, pp.615-619, 2002.

[17] M. J. Jones and J. M. Rehg. Statistical color models with application to skin detection. Technical Report CRL 98/11, Compaq, 1998.

[18] J. C. Terrillon, M. David, and S. Akamatsu. Automatic detection of human faces in natural scene images by use of a skin color model and of inavariant moments. In IEEE Third International Conference on Automatic Face and gestur Recognition, pages 112-117, 1998

[19] M. Jones and J. M. Rehg. Statistical color models with application

to skin detection. In Computer Vision and Pattern Recognition, pages 274-280, 1999.

[20] G. Wyszecki and W.S. Styles. Color Science: Concepts and Methods, Quantitative Data and Formulae, Second Edition, John Wiley \& Sons, New York, 1982.
[21] Chang H., and Robes U. " face Detection, May $2000 \quad$ http://www-csstudents.stanford.edu/ robles/ee368/main.html

[22] Terrillon J.C., Shirazi, M. N., Fukamachi, H., and Akamastu "Comparative performance of different skin chrominance models and chrominance spaces for the automatic detection of human faces in color images." In Proc. of the International Conference on Face and Gesture Recognition, 54-61,2000 